\title{
Minocycline Alleviates Abnormal Phagocytosis of Synapses by Microglia in a Mouse Model of Depression
}

\section{Qiu-Qin Han ( $\sim$ hqq@sumhs.edu.cn )}

Shanghai University of Medicine and Health Sciences

\section{Shi-Yu Shen}

Fudan University

\section{Xiao-Rong Chen}

Changzhi Medical College

\section{Adam Pilot}

Beijing University of Chinese Medicine

\section{Ling-Feng Liang}

Fudan University

Jia-Rui Zhang

Fudan University

\section{Wen-Hui Li}

Shanghai University of Medicine and Health Sciences

Yi Fu

Shanghai University of Medicine and Health Sciences

Jia-Mei Le

Shanghai University of Medicine and Health Sciences

\section{Pei-Qing Chen}

Shanghai University of Medicine and Health Sciences Jin Yu

Fudan University

\section{Research}

Keywords: depression, CSDS, microglia, synapse, phagocytosis, minocycline

Posted Date: October 12th, 2021

DOI: https://doi.org/10.21203/rs.3.rs-955145/v1 
License: (c) (i) This work is licensed under a Creative Commons Attribution 4.0 International License. Read Full License 


\section{Abstract \\ Background}

Depression is an affective disorder characterized by low mood and loss of interest. So far, the mechanism of antidepressants commonly used in clinical practice has proved problematic, thus it is urgent to gain an updated understanding of the pathogenesis of depression and find potential therapeutic targets. As both functional brain imaging studies and autopsy reports indicated that there is indeed a loss of synapses in depressed patients, it is necessary to explore the mechanism of this process.

\section{Methods}

We firstly investigated the effect of CSDS (a mouse model of depression) on behaviors, synapses, microglia, and phagocytosis of synapses by microglia in mice. Then, to confirm the role of microglia in depression, we used minocycline, a microglial activation inhibitor, to study its effect on behaviors and phagocytosis of synapses in stressed mice.

\section{Results}

Our results show that the expression levels of PSD-95 in the hippocampus of CSDS-induced depression mice are significantly reduced, while the microglia are significantly activated. We co-labeled the synaptic protein PSD-95 with the microglia marker Iba-1, and found that the microglia in the hippocampus of stressed mice contained significantly more PSD-95 engulfed puncta, which revealed that microglia in stressed mice abnormally phagocytized synapses. Moreover, our results indicated that minocycline treatment dampened microglial activation, reduced synaptic loss, alleviated behavioral impairment, and reduced abnormal phagocytosis of synapses by microglia in stressed mice.

\section{Conclusions}

Under depressive pathological conditions, the activated microglia may abnormally engulf neuronal synapses causing synaptic loss. Our findings are important for the discovery of novel drugs for the treatment of depression.

\section{Background}

Depression is an affective disorder characterized by low mood and loss of interest. With the continuous development and progress of society, social stress is gradually increasing, and the prevalence of depression is increasing year by year, which has become a serious social public health problem[1]. According to a recent study published in Lancet Psychiatry, the lifetime prevalence of depression in China is $6.8 \%$, of which major depressive disorder (MDD) is 3.4\%[2]. For a long time, the monoamine transmitter 
hypothesis has played a dominant role in the study of the pathogenesis of depression. At present, the mechanism of commonly used antidepressants in the clinic is mainly based on the monoamine transmitter hypothesis. However, this approach has shown multiple problems, such as large individual differences in curative effect, low effective rate, high incidence of side effects, delayed drug effect, and low patient compliance rate, serious withdrawal symptoms[3, 4]. In view of the many problems of clinically available antidepressants, it is assumed that in addition to changes in the monoamine transmitter system, there are other dysfunctions in depression. Indeed, the pathogenesis of depression is complex and diverse, and has not been precisely clarified, thus it is urgent to unravel the pathogenesis of depression anew and find potential therapeutic targets.

Neuronal synapses are mainly composed of presynaptic structures, synaptic gaps, and postsynaptic structures. It is the structural basis of information transmission between neurons and is a key part of neuroplasticity regulation. Functional brain imaging studies[5] reported that synaptic connections in the hippocampus, prefrontal cortex, and amygdala in patients with depression were significantly reduced, suggesting that neuronal synapses may have been lost in depressed patients. In addition, an autopsy report[6] indicated that synaptic structures and function-related genes in the hippocampus of depressed patients were significantly reduced, including synaptosomal-associated protein 25 (SNAP25) and glutamate receptors. Another autopsy report[7] stated that post-synaptic density-95 (PSD-95) and $\mathrm{Y}^{-}$ aminobutyric acid receptors in the prefrontal cortex of patients with depression were also significantly reduced. Both autopsy reports suggest that there is indeed a loss of synapses in the brains of patients with depression. Researchers have also observed similar experimental results in animal models of depression, where the expressions of PSD-95, Synaptophysin, and growth-associated protein 43 in the hippocampus and prefrontal cortex of depressed rats induced by mother-infant separation were significantly reduced[8]. Our group[9] also found that the dendritic spine density in the hippocampus of depressed mice, induced by chronic unpredictable mild stress, (CUMS) was significantly reduced. In addition, some drugs, such as Liraglutide[10], Calpeptin[11], and Panaxynol[12] are able to upregulate the expression of synaptophysin, PSD-95, and synapsin I in the hippocampus, or enhance the hippocampal nerve metasynaptic plasticity and long-term potentiation (LTP) to alleviate depressive behaviors of model animals. These results suggest that reversing the loss of synapses can improve depressive behaviors of model animals. In view of this, it is necessary to explore the mechanism of synapse loss, which may provide a new strategy for the treatment of depression.

Shiosaka et al. proposed the concept of "synaptic microenvironment"[13]. Synaptic microenvironment refers to the synapses of neurons and their internal and external environments, including microglia, astrocytes, oligodendrocytes, vascular endothelial cells, extracellular matrix, and cytokines. The synaptic microenvironment has an important influence on the structure and function of synapses. Therefore, paying attention to the changes of various cells or factors in the synaptic microenvironment can provide new ideas for exploring the mechanism of synaptic loss in depression. Microglia are innate immune cells in the central nervous system, accounting for about $10-12 \%$ of adult brain cells, and are one of the important components of the synaptic microenvironment. Under normal circumstances, the main role of microglia is to remove pathogens and phagocyte debris. In addition, microglia also monitor and trim the 
synapses through phagocytosis to promote the maturation and maintain the function of the synapses[14]. In 2011, Paolicelli RC et al.[15] described in their Science article the finding, that normal mouse microglia contained synaptic fragments. These fragments were synapse-related molecules, including the presynaptic protein SNAP25 and the postsynaptic protein PSD-95, which confirmed the phagocytosis of synapses by glial cells. It is thus rendered obvious, that the loss of neuronal synapses is closely related to the phagocytosis of microglia.

One positron emission tomography (PET) study[16] reported that translocator protein (TSPO), a marker of microglia was significantly upregulated in the hippocampus, frontal cortex, and temporal cortex of patients with depression, suggesting that microglial activation may be involved in the pathogenesis of depression. In addition, Du Preez A et al. [17] found that microglia in the hippocampal dentate gyrus of CUMS depression mice were significantly activated. Our group performed PET imaging and immunohistochemical studies[18], and found that TSPO in the hippocampus of depressed rats was significantly upregulated, and the density of microglia increased significantly. Thus, we asked if under pathological conditions, in addition to participating in neuroinflammation, activated microglia can abnormally engulf neuronal synapses.

We speculated that the loss of synapses in the hippocampus of patients with depression may also be caused by the abnormal phagocytosis of activated microglia. In order to verify this conjecture, we conducted two experiments: (1) As shown in Figure 1A, we investigated the effect of chronic social defeat stress (CSDS) on behaviors, synapses, microglia, and phagocytosis of synapses by microglia in mice; (2) As shown in Figure 4A, to confirm the role of microglia in depression, we investigated the effect of minocycline (a microglial activation inhibitor) on behaviors and phagocytosis of synapses in stressed mice. Our work thus aims to explicate the role of microglia phagocytosis of synapses in the pathogenesis of depression for the first time, in order to provide new insights into the pathogenesis of depression and its treatment.

\section{Methods}

\subsection{Animals}

For all experiments, we used male C57BL/6J mice (7-8 weeks, from Vital River Laboratories, Beijing, China). The animals were provided with food and water (normal diet) available ad libitum and were allowed a one-week habituation period before experimental manipulation, while being housed at $23 \pm 2{ }^{\circ} \mathrm{C}$ on a $12 \mathrm{~h}$ light/dark cycle (lights on at 7:00 A.M.). CD-1 retired breeders (male, 8-9 months, from Vital River Laboratories, Beijing, China) were used as the aggressors. This study was carried out in accordance with the National Institute of Health Guide for the Care and Use of Laboratory Animals.

\subsection{Chronic social defeat stress (CSDS)}

The CSDS procedure was performed as previously described[19, 20, 21]. The C57BL/6J mice were individually introduced to the home cage of an unfamiliar aggressive CD-1 resident mouse for 5-10 min 
and exposed to physical defeat, after which it was housed together with the CD-1, but separated by a perforated plastic divider to allow for visual, olfactory and auditory contact for the remainder of the $24 \mathrm{~h}$. The mice were subject to social defeat for 10 consecutive days, each day being exposed to a different CD-1 mouse.

\subsection{Social interaction test (SIT)}

The SIT was performed on day 11 , as previously described $[19,20,21]$. This test was performed in a clean open arena $(42 \mathrm{~cm} \times 42 \mathrm{~cm}$ ). Each time, it consisted of two $2.5 \mathrm{~min}$ sessions. In the first (also called NoTarget) session, a C57BL/6J mouse was introduced to the arena with an empty mesh cage $(10 \mathrm{~cm} \times 6$ $\mathrm{cm}$ ) at one of its sides. In the second (Target) session, a mesh cage with an unfamiliar CD-1 mouse replaced the empty cage. The area of $26 \mathrm{~cm} \times 14 \mathrm{~cm}$, meaning $8 \mathrm{~cm}$ around the mesh cage, was defined as the interaction zone. The time spent in the interaction zone was measured, and the social interaction rate was defined as the time spent in the interaction zone with the aggressor present (Target) divided by the time spent in the interaction zone without the aggressor (No-Target).

\subsection{Open field test (OFT)}

OFT was performed as previously described[21], and was performed in dim light (15 lux). The OFT apparatus consisted of a square open box $(50 \mathrm{~cm} \times 50 \mathrm{~cm} \times 40 \mathrm{~cm})$. Mice were individually placed in the center of the arena and allowed to explore for $5 \mathrm{~min}$, while their activity was video-tracked. Total distance traveled, as well as the time spent and distance traveled in the center-zone of the box were recorded. The locomotor activity was tracked by a video recorder and analyzed using specialized software (Shanghai Mobile Datum Information Technology Company, Shanghai, China).

\subsection{Elevated plus maze test (EPM)}

Every EPM test was performed 3 hours after the OFT, and in a way previously described[21]. It took place in dim light (15 lux). The EPM is an apparatus elevated $40 \mathrm{~cm}$ above the floor and divided into two open and two closed arms $(5 \mathrm{~cm} \times 30 \mathrm{~cm})$. The closed arms have $20-\mathrm{cm}$-high walls, while the open arms do not have boundaries. Mice were placed in the center of the maze facing an open arm, and allowed to freely explore for $5 \mathrm{~min}$, while their activity was video-tracked and analyzed by software (Shanghai Mobile Datum Information Technology Company, Shanghai, China). Open-Arm Time \% was calculated as [time in open arms]/[time in all arms] $\times 100$.

\subsection{Forced swimming test (FST)}

The FST[21] was performed in a clear glass cylinder (height $32 \mathrm{~cm}$, diameter $16 \mathrm{~cm}$ ), filled with $15 \mathrm{~cm}$ of water $\left(23-25^{\circ} \mathrm{C}\right)$. The test lasted for $5 \mathrm{~min}$, during which the duration of immobility was recorded with a stopwatch by a researcher blind to the grouping of the experimental mice. Immobility is defined as passive floating or making minimal movements necessary to keep the nostrils above water.

\subsection{Immunohistochemistry}


Immunohistochemistry was performed as previously described[21]. The primary antibodies included antiPSD-95 antibody (1:200, ABCAM, ab192757), anti-Synaptophysin antibody (1:200, ABCAM, ab32127) and anti-Ibal-1 antibody (1:500, Wako, 019-19741). The sections were observed using a confocal laser scanning microscope (model FV1000, Olympus).

\subsection{Western blotting}

After decapitation, the hippocampi were quickly dissected and stored at $-80^{\circ} \mathrm{C}$ until use. The brain tissues were processed as previously described[21]. The primary antibodies included anti-lbal-1 antibody (1:1000, ABCAM, ab5076), and anti- $\beta$-actin antibody (1:1000, CST, \#12620). Western blot images were captured using an ImageQuant LAS4000 Mini Image Analyzer (GE Healthcare, Buckinghamshire, UK) and the band levels were quantified using Quantity One, version 4.62 .

\subsection{Intraperitoneal drug administration}

Minocycline (Cat: M22254) was obtained from ABCONE (Shanghai, P.R. China). Along with the social defeat stress procedure, control mice and defeated mice were daily injected with minocycline $(50 \mathrm{mg} / \mathrm{kg})$ or its vehicle $(0.9 \% \mathrm{NaCl})$ intraperitoneally.

\subsection{Statistical analysis}

The data are presented as the mean \pm standard error (SEM). All statistical analyses were performed using GraphPad Prism 6.01 (GraphPad Software Inc., San Diego, CA). The statistical significance of differences between groups was analyzed using a T-test or two-way analysis of variance (ANOVA). The statistical significance level was set to $p<0.05$. The graphs were plotted using GraphPad Prism 6.01.

\subsection{Experimental design}

\subsubsection{Experiment 1: Effect of CSDS on behaviors, synapses, microglia, and phagocytosis of synapses by microglia in mice}

To investigate the effect of CSDS on behaviors, synapses, microglia, and phagocytosis of synapses by microglia in mice (Figure 1A), the first experiment was performed. Mice were allowed a one-week habituation period before any experimental manipulation. Then, the mice were divided into two groups: control group (No CSDS) and CSDS group. CSDS mice were exposed to ten days of CSDS. On day 11, the SIT, OFT and EPM were conducted. On day 12, the FST was performed. On day 13, the mice were sacrificed. Then, the synapses, microglia, and phagocytosis of synapses by microglia in hippocampi were examined.

\subsubsection{Experiment 2: Effect of minocycline (a microglial activation inhibitor) on behaviors and phagocytosis of synapses by microglia in stressed mice}

To illustrate the effect of minocycline on behaviors and phagocytosis of synapses by microglia, we administered the control and stressed mice with minocycline or its vehicle by intraperitoneal injection (Figure 4A). Mice were allowed a one-week habituation period before any experimental manipulation. 
Then, mice were divided into four groups: No CSDS + Saline group, No CSDS + minocycline group, CSDS + Saline group, and CSDS + minocycline group. CSDS mice were exposed to ten days of CSDS. During that time minocycline or its vehicle were administered. On day 11, the SIT, OFT and EPM were performed. On day 12 , the FST was conducted. On day 13, the mice were sacrificed and the phagocytosis of synapses by microglia in their hippocampi was detected.

\section{Results}

\subsection{CSDS induced depressive-like behaviors in mice}

The depression state of mice was successfully achieved through the CSDS model (Figure 1A). As shown in Figure 1, compared with the control mice, the social interaction rate of CSDS mice in the SIT was significantly reduced (Figure 1B), the immobility time in the FST was markedly increased (Figure 1C), and the time percentage spent in the open arms in the EPM was significantly reduced (Figure 1D). In the OFT, although the total distance of the model mice was not different from the control mice, the time spent and distance traveled in the center-zone were both significantly reduced (Figure 1E-G). These results demonstrate that CSDS induced significant depressive-like behaviors in mice.

\subsection{CSDS induced microglial activation and synaptic loss in mice}

As shown in Figure 2A-C, the relative intensity and the soma size of microglia in the hippocampus of the stressed mice were significantly increased, compared with control mice. Western blotting results also showed that the protein levels of Iba1 were significantly increased in the stressed mice (Figure 2D, E). These results indicate that CSDS induced microglial activation in stressed mice. Besides, the expression levels of PSD-95 (Figure 2F, G) and synaptophysin (Figure 2H, I) in the hippocampus of stressed mice were significantly reduced. The above results indicate that CSDS caused both synaptic loss as well as microglial activation in model mice. Since microglia can phagocytize synapses, we put forward the following question: Is the synaptic loss caused by abnormal activation of microglia in stressed mice?

\subsection{CSDS induced abnormal phagocytosis of synapses by microglia in mice}

To clarify, whether the synaptic loss was caused by the abnormal activation of microglia in stressed mice, we co-labeled the synaptic PSD-95 or synaptophysin with the microglia marker lba-1 respectively. Compared with the control mice, the microglia in the hippocampus of stressed mice contained significantly more PSD-95 engulfed puncta (Figure 3A, B), which revealed that microglia in stressed mice abnormally phagocytized synapses. However, we couldn't find any synaptophysin in microglia of control or stressed mice. The reason for that could be that synaptophysin is unstable and easily degraded in the process of phagocytosis by microglia. To further confirm the role of microglia in depression, we used a microglial activation inhibitor to study its effect on behaviors and phagocytosis of synapses in stressed mice. 


\subsection{Minocycline treatment dampened microglial activation in stressed mice}

Minocycline, as a microglial activation inhibitor, was used to assess the role of microglial activation in CSDS-induced abnormal behaviors and pathology. To illustrate the role of microglial activation in stressed mice, we administered the control and stressed mice with minocycline or vehicle by intraperitoneal injection (Figure 4A). As expected, minocycline treatment significantly dampened the increased intensity and soma size of microglia in the stressed mice (Figure 4B-D). Western blotting results also showed that the protein levels of Iba1 were significantly reduced in stressed mice treated with minocycline (Figure 4E, F). These results indicate that minocycline treatment dampened microglial activation in stressed mice.

\subsection{Minocycline treatment alleviated synaptic loss and behavioral impairments in stressed mice}

As shown in Figure 5, chronic treatment with minocycline significantly reversed the reduced levels of PSD95 (Figure 5A, B) and synaptophysin (Figure 5C, D) in the hippocampus of stressed mice. Besides, minocycline treatment normalized the behavioral deficits of stressed mice, as indicated by normal social interaction rate in the SIT (Figure 6A), normal immobility time in the FST (Figure 6B), normal time percentage spent in the open arms in the EPM (Figure $6 \mathrm{C}$ ), and normal time spent and distance traveled in the center-zone in the OFT (Figure 6D-F).

\subsection{Minocycline treatment reduced abnormal phagocytosis of synapses by microglia in stressed mice}

The immunohistochemical co-labelling experiment revealed that minocycline treatment significantly reduced the number of PSD-95 engulfed puncta contained in microglia in the hippocampus of stressed mice (Figure 7A, B), which hints that minocycline treatment reduced abnormal phagocytosis of synapses by microglia in stressed mice.

\section{Discussion}

As both functional brain imaging studies[5] and autopsy reports[6][7] indicated that there is indeed a loss of synapses in the brains of patients with depression, it is necessary to explore the mechanism of synapse loss, which may provide a new strategy for the treatment of depression. In this study, the results showed that the expression levels of PSD-95 and synaptophysin in the hippocampus of CSDS-induced depression mice are significantly reduced, while the microglia are significantly activated. Microglia are the major immune cells in the central nervous system and they control the inflammation in brain through releasing inflammatory cytokines, clearing apoptotic cells, regulating synaptic plasticity and synapse pruning[22]. As microglia, the brain's specialized phagocytes, can eliminate synapses[23], we proposed that the synaptic loss may be caused by the abnormal activation of microglia in stressed mice. In order to 
verify this conjecture, we co-labeled the synaptic protein PSD-95 with the microglia marker Iba-1, and found that compared with the control mice, the microglia in the hippocampus of stressed mice contained significantly more PSD-95 engulfed puncta, which revealed that microglia in stressed mice abnormally phagocytized synapses. To confirm the role of microglia in depression, we used minocycline, a microglial activation inhibitor, to study its effect on behaviors and phagocytosis of synapses in stressed mice. The results indicated that minocycline treatment dampened microglial activation, reduced synaptic loss and alleviated behavioral impairments in stressed mice. In addition, minocycline treatment reduced abnormal phagocytosis of synapses by microglia in stressed mice. Thus, under depressive pathological conditions, the activated microglia may abnormally engulf neuronal synapses causing synaptic loss.

This study proposed for the first time the role of microglia's phagocytosis of synapses in the pathogenesis of depression, which is innovative. However, the mechanism of microglial synaptic phagocytosis in depression is still not clear. There are several possible mechanisms: (1) One study indicated the absence of triggering receptor expression on myeloid cells 2 (TREM2) in microglia resulted in impaired synapse elimination in Trem2(-/-) mice, thus TREM2 might be essential for microgliamediated synaptic refinement [24]; (2) Another study revealed that transforming growth factor (TGF)-beta may regulate neuronal C1q expression to initiate C1q/C3/CR3 (CD11b) complement- and microgliamediated synaptic pruning. Besides, mice lacking TGF-beta receptor II (TGFbetaRII) in retinal neurons were found to have reduced C1q expression and reduced synaptic localization of complement[25]; (3) Yet another study showed that REV-ERBalpha deletion increased synapse loss and phagocytosis of synapses by microglia in the CA3 region of the hippocampus. It was found that REV-ERBalpha mediated complement expression and diurnal regulation of phagocytosis of synapses by microglia[26]. Thus, the mechanism of dysfunctional molecular interaction between microglia and synapses in depression needs to be studied further.

\section{Conclusions}

Taken together, we conclude, that under depressive pathological conditions, the activated microglia may abnormally engulf neuronal synapses causing synaptic loss. However, the mechanism of dysfunctional molecular interaction between microglia and synapses in depression has not been found yet. In spite of this limitation, our study adds to the accumulating evidence, that microglial activation is involved in the pathogenesis of depression. One of the major strengths of this study is that it provides insight into the role of abnormal phagocytosis of synapses by microglia in depression. Undeniably, our findings are important for the discovery of novel drugs for the treatment of depression.

\section{List Of Abbreviations}

Major depressive disorder (MDD)

Post-synaptic density-95 (PSD-95) 
Chronic unpredictable mild stress (CUMS)

Chronic social defeat stress (CSDS)

Social interaction test (SIT)

Open field test (OFT)

Elevated plus maze test (EPM)

Forced swimming test (FST)

\section{Declarations}

\section{Ethics approval and consent to participate}

This study was approved by the Animal Ethics Committee of Shanghai University of Medicine and Health Sciences.

\section{Consent for publication}

Not applicable.

\section{Availability of data and material}

The datasets during and/or analysed during the current study available from the corresponding author on reasonable request.

\section{Competing interests}

The authors declare no conflict of interest.

\section{Funding}

This study was funded by grants from Construction project of Shanghai Key Laboratory of Molecular Imaging (18DZ2260400), the Key Program of National Natural Science Foundation of China (Grant No.81830052), the National Natural Science Foundation of China (Grant No.81903961), and the Schoollevel Fund (E3-0200-21-201011-73) of Shanghai University of Medicine and Health Sciences.

\section{Authors' contributions}

Jin Yu, Qiu-Qin Han, and Xiao-Rong Chen designed this experiment. Shi-Yu Shen, Ling-Feng Liang, and Jia-Rui Zhang performed the behavior tests. Wen-Hui Li, Yi Fu, Jia-Mei Le, and Pei-Qing Chen conducted the biochemical experiments. Adam Pilot wrote and revised this manuscript.

\section{Acknowledgements}


Not applicable.

\section{References}

1. Steffen A, Thom J, Jacobi F, Holstiege J, Batzing J. Trends in prevalence of depression in Germany between 2009 and 2017 based on nationwide ambulatory claims data. J Affect Disord. 2020;271:239-47.

2. Huang Y, Wang Y, Wang H, Liu Z, Yu X, Yan J, et al. Prevalence of mental disorders in China: a crosssectional epidemiological study. Lancet Psychiat. 2019;6:211-24.

3. Kaster MP, Moretti M, Cunha MP, Rodrigues AL. Novel approaches for the management of depressive disorders. Eur J Pharmacol. 2016;771:236-40.

4. Kendrick T. Strategies to reduce use of antidepressants. Br J Clin Pharmacol (2020).

5. Zeng LL, Shen H, Liu L, Wang L, Li B, Fang P, et al. Identifying major depression using whole-brain functional connectivity: a multivariate pattern analysis. Brain. 2012;135:1498-507.

6. Duric V, Banasr M, Stockmeier CA, Simen AA, Newton SS, Overholser JC, et al. Altered expression of synapse and glutamate related genes in post-mortem hippocampus of depressed subjects. Int $\mathrm{J}$ Neuropsychopharmacol. 2013;16:69-82.

7. Zhao J, Bao AM, Qi XR, Kamphuis W, Luchetti S, Lou JS, et al. Gene expression of GABA and glutamate pathway markers in the prefrontal cortex of non-suicidal elderly depressed patients. $J$ Affect Disord. 2012;138:494-502.

8. Cui Y, Cao K, Lin H, Cui S, Shen C, Wen W, et al. Early-Life Stress Induces Depression-Like Behavior and Synaptic-Plasticity Changes in a Maternal Separation Rat Model: Gender Difference and Metabolomics Study. Front Pharmacol. 2020;11:102.

9. Huang HJ, Chen XR, Han QQ, Wang J, Pilot A, Yu R, et al. The protective effects of Ghrelin/GHSR on hippocampal neurogenesis in CUMS mice. Neuropharmacology. 2019;155:31-43.

10. Weina H, Yuhu N, Christian H, Birong L, Feiyu S, Le W. Liraglutide attenuates the depressive- and anxiety-like behaviour in the corticosterone induced depression model via improving hippocampal neural plasticity. Brain Res. 2018;1694:55-62.

11. Song Z, Shen F, Zhang Z, Wu S, Zhu G. Calpain inhibition ameliorates depression-like behaviors by reducing inflammation and promoting synaptic protein expression in the hippocampus. Neuropharmacology. 2020;174:108175.

12. Sun X, Zhang T, Zhao Y, Cai E, Zhu H, Liu S. Panaxynol attenuates CUMS-induced anxiety and depressive-like behaviors via regulating neurotransmitters, synapses and the HPA axis in mice. Food Funct. 2020;11:1235-44.

13. Shiosaka S, Yoshida S. Synaptic microenvironments--structural plasticity, adhesion molecules, proteases and their inhibitors. Neurosci Res. 2000;37:85-9.

14. Graeber MB. Changing face of microglia. Science. 2010;330:783-8. 
15. Paolicelli RC, Bolasco G, Pagani F, Maggi L, Scianni M, Panzanelli P, et al. Synaptic pruning by microglia is necessary for normal brain development. Science. 2011;333:1456-8.

16. $\mathrm{Li} \mathrm{H}$, Sagar AP, Keri S. Microglial markers in the frontal cortex are related to cognitive dysfunctions in major depressive disorder. J Affect Disord. 2018;241:305-10.

17. Du Preez A, Onorato D, Eiben I, Musaelyan K, Egeland M, Zunszain PA, et al. Chronic stress followed by social isolation promotes depressive-like behaviour, alters microglial and astrocyte biology and reduces hippocampal neurogenesis in male mice. Brain Behav Immun. 2021;91:24-47.

18. Wang YL, Han QQ, Gong WQ, Pan DH, Wang LZ, Hu W, et al. Microglial activation mediates chronic mild stress-induced depressive- and anxiety-like behavior in adult rats. J Neuroinflammation. 2018;15:21.

19. Han Q, Yang L, Liu Y, Lv N, Yu J, Wu G, et al. Resiliency to social defeat stress relates to the interstrain social interaction and is influenced by season variation. Neurosci Lett. 2014;561:13-7.

20. Han QQ, Yang L, Huang HJ, Wang YL, Yu R, Wang J, et al. Differential GR Expression and Translocation in the Hippocampus Mediates Susceptibility vs. Resilience to Chronic Social Defeat Stress. Front Neurosci. 2017;11:287.

21. Han QQ, Huang HJ, Wang YL, Yang L, Pilot A, Zhu XC, et al. Ghrelin exhibited antidepressant and anxiolytic effect via the p38-MAPK signaling pathway in hippocampus. Prog Neuropsychopharmacol Biol Psychiatry. 2019;93:11-20.

22. Deng SL, Chen JG, Wang F. Microglia: A Central Player in Depression. Curr Med Sci. 2020;40:391400.

23. Brown GC, Neher JJ. Microglial phagocytosis of live neurons. Nat Rev Neurosci. 2014;15:209-16.

24. Filipello F, Morini R, Corradini I, Zerbi V, Canzi A, Michalski B, et al. The Microglial Innate Immune Receptor TREM2 Is Required for Synapse Elimination and Normal Brain Connectivity. Immunity. 2018;48:979-91.e8.

25. Bialas AR, Stevens B. TGF-beta signaling regulates neuronal C1q expression and developmental synaptic refinement. Nat Neurosci. 2013;16:1773-82.

26. Griffin P, Sheehan PW, Dimitry JM, Guo C, Kanan MF, Lee J, et al., REV-ERBalpha mediates complement expression and diurnal regulation of microglial synaptic phagocytosis. Elife 9 (2020).

\section{Figures}


A

Experimental group

Control

CSDS

$\begin{array}{llll}\text { Habitutation } & \text { CSDS } & \text { Behavioral tests }>\text { S } \\ \text { B SIT } & C & \text { FST } & \text { D }\end{array}$
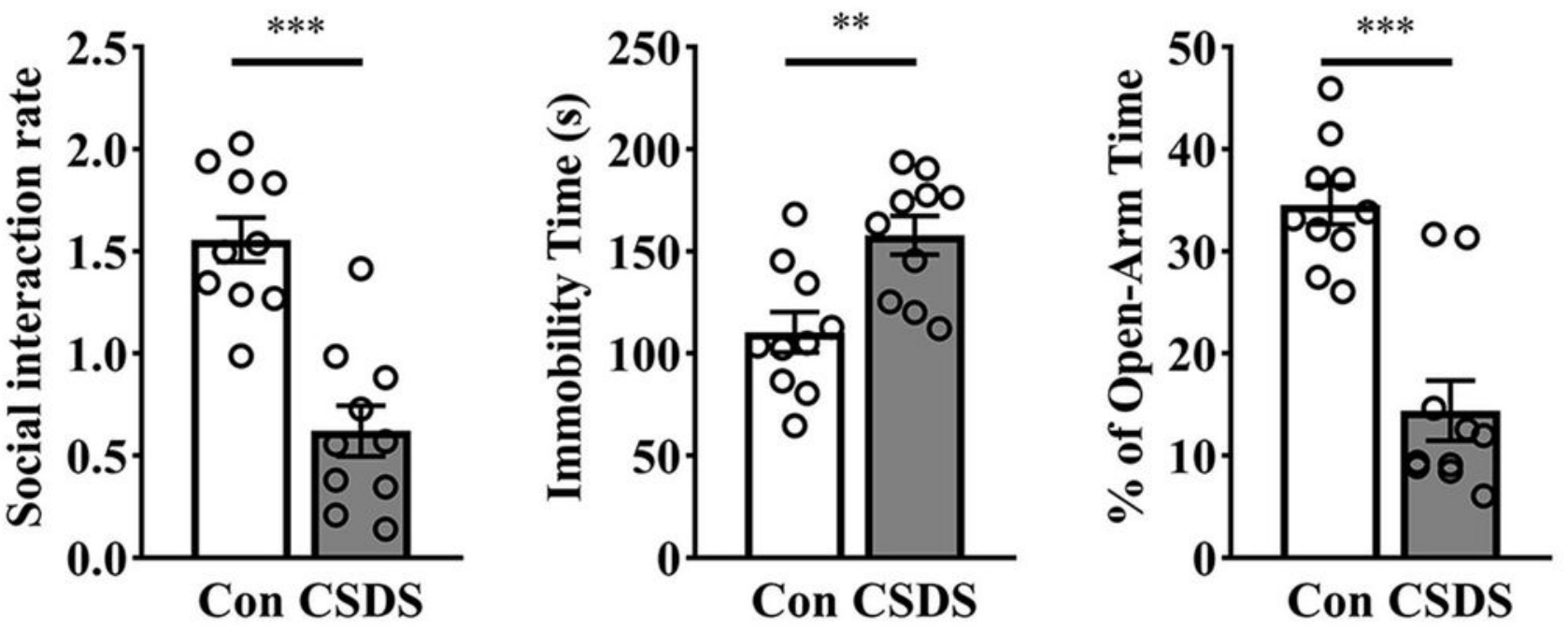

E

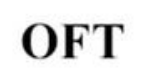

$\mathrm{F}$

OFT

G

OFT
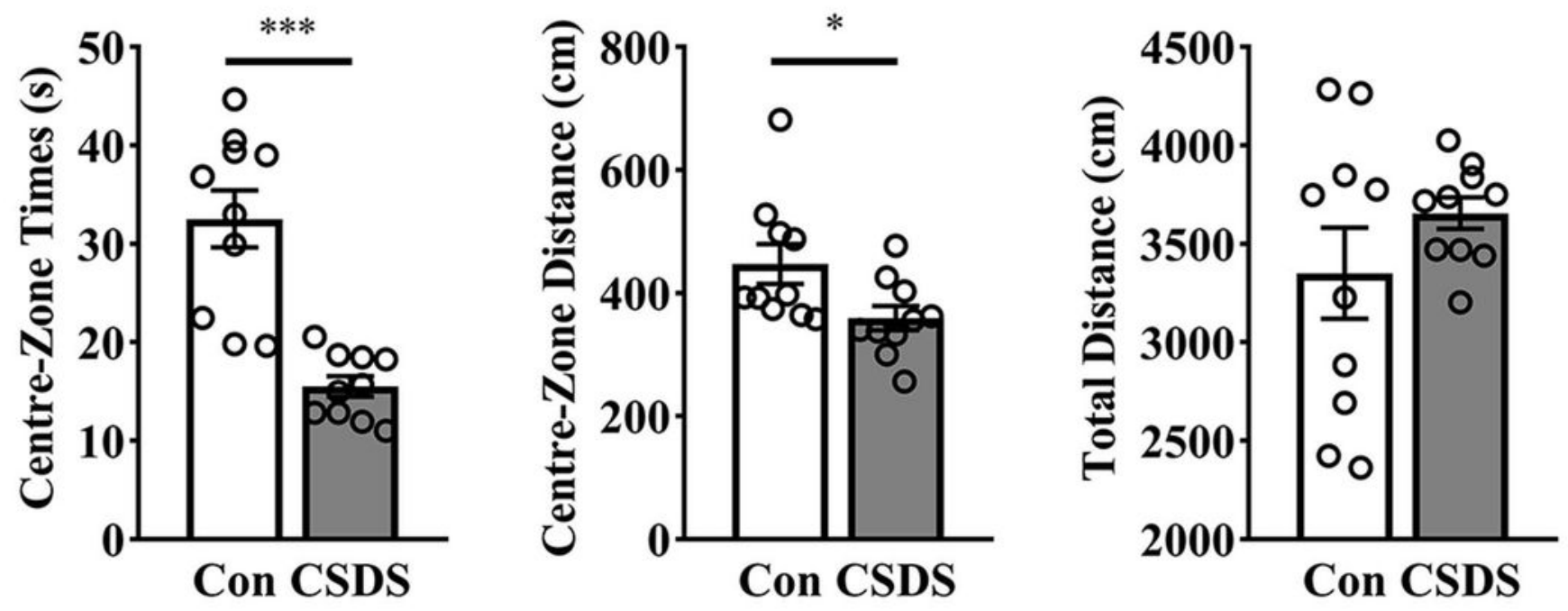

Figure 1

CSDS induced depressive-like behavior in mice. A, Experimental procedure. B, Social interaction ratio in the social interaction test. C, Immobility time in the forced swimming test. D, Ratio of time spent in the open arms in the elevated plus maze test. E, Time spent in the center-zone in the open field test. F, Distance traveled in the center-zone in the open field test. G, Total distance traveled in the open field test. 
$\mathrm{N}=10$ /group. All data are shown as mean \pm SEM. ${ }^{\star} \mathrm{P}<0.05,{ }^{*} \mathrm{P}<0.01,{ }^{*} * \mathrm{P}<0.001$. Con: Control; CSDS: Chronic social defeat stress.
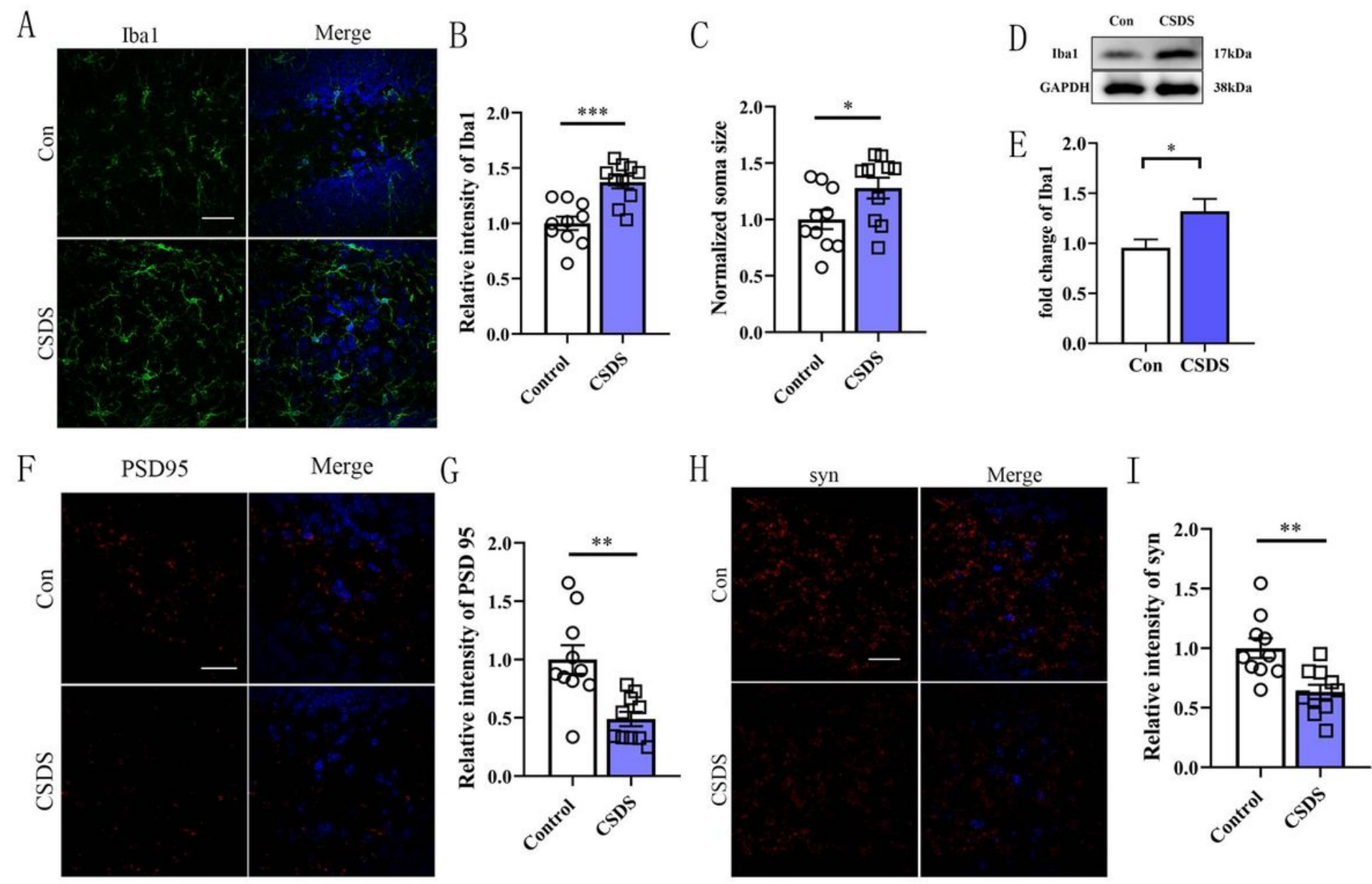

Figure 2

CSDS induced microglial activation and synaptic loss in mice. A, Representative immunofluorescence staining for Ibal in the hippocampus. B, Quantitative analysis of the relative intensity of Iba1. C, Quantitative analysis of the soma size of microglia. D, Representative western blot image for Ibal in the hippocampus. E, Quantitative western blot analysis of the relative intensity of Iba1. F, Representative immunofluorescence staining for PSD-95 in the hippocampus. G, Quantitative analysis of the relative intensity of PSD-95. H, Representative immunofluorescence staining for synaptophysin in the hippocampus. I, Quantitative analysis of the relative intensity of synaptophysin. $N=10 /$ group. All data are shown as mean \pm SEM. ${ }^{*} P<0.05,{ }^{*} \mathrm{P}<0.01,{ }^{\star * *} \mathrm{P}<0.001$. CSDS: Chronic social defeat stress. 


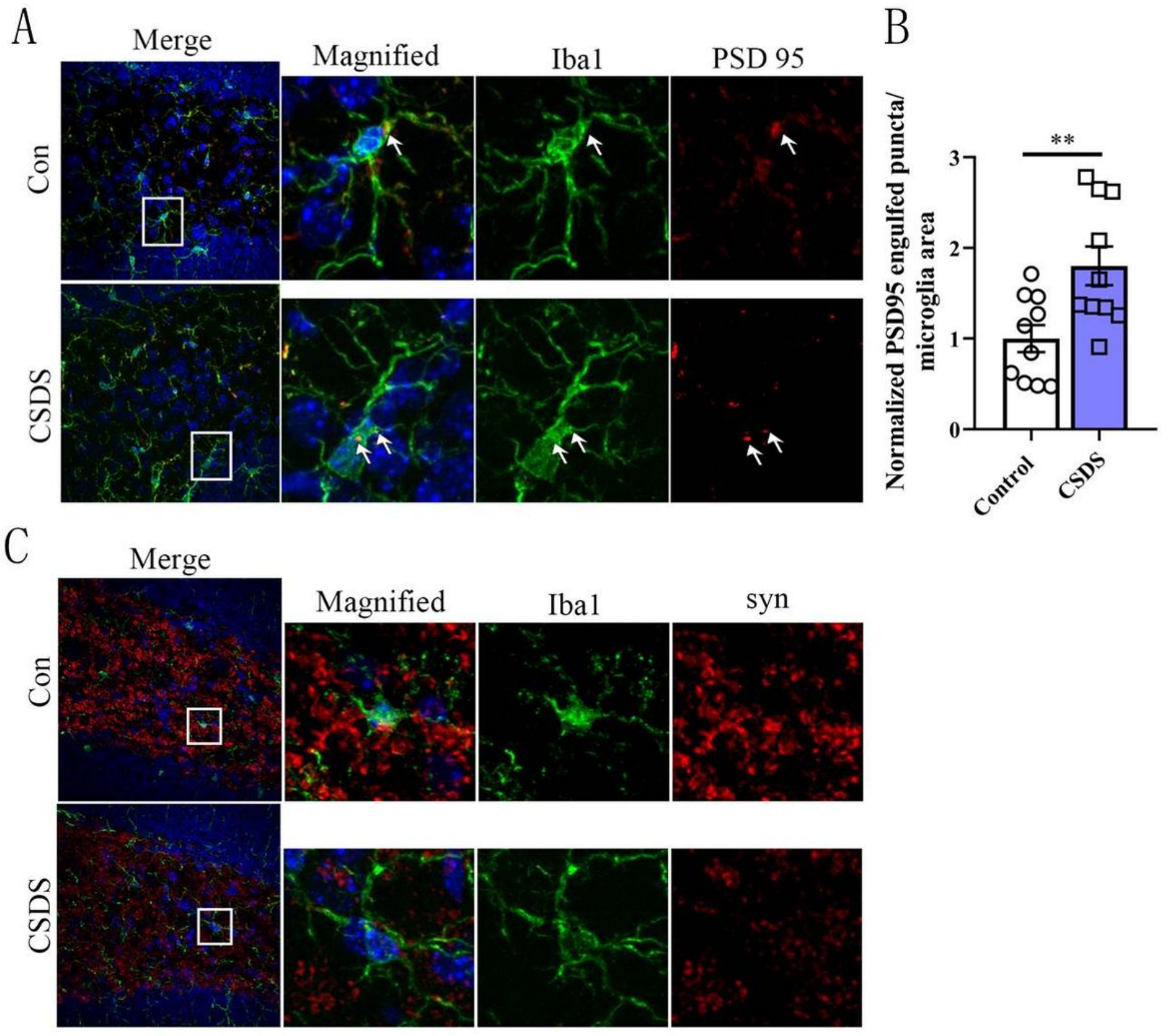

Figure 3

CSDS induced abnormal phagocytosis of synapses by microglia in mice. A, Representative immunofluorescence staining for PSD-95 co-labeled with lbal in the hippocampus. B, Quantitative analysis of the PSD-95 engulfed puncta in microglia. C, Representative immunofluorescence staining for synaptophysin co-labeled with lbal in the hippocampus. $\mathrm{N}=10$ /group. All data are shown as mean $\pm \mathrm{SEM}$. $\star \star P<0.01$. CSDS: Chronic social defeat stress. 


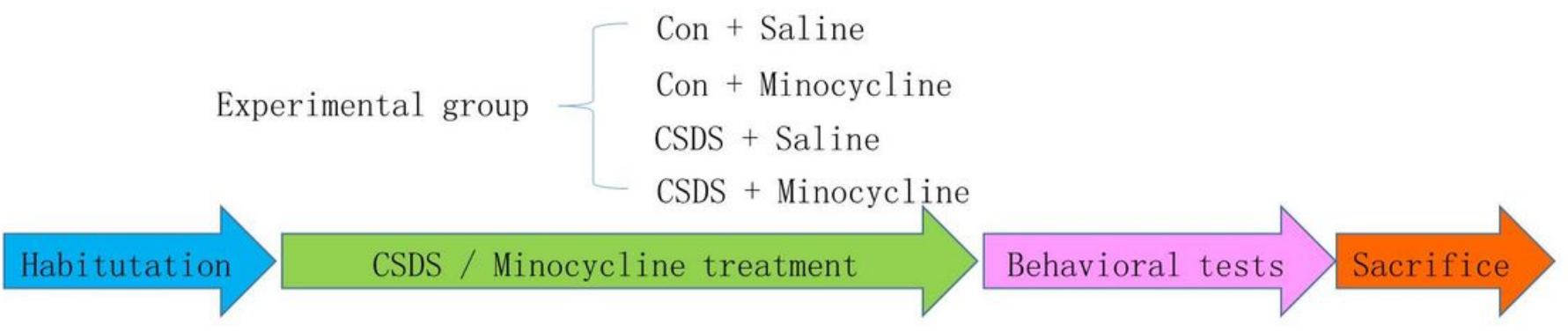

B
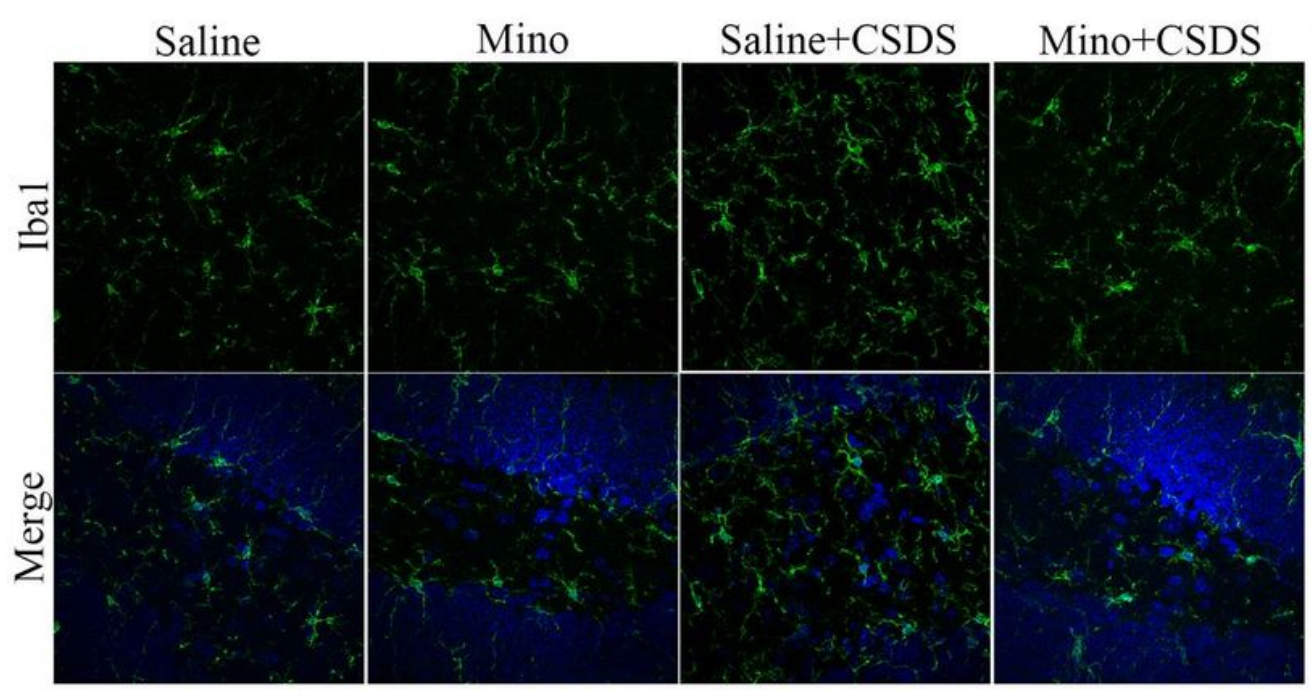

C ○ Control $\square$ csps

E

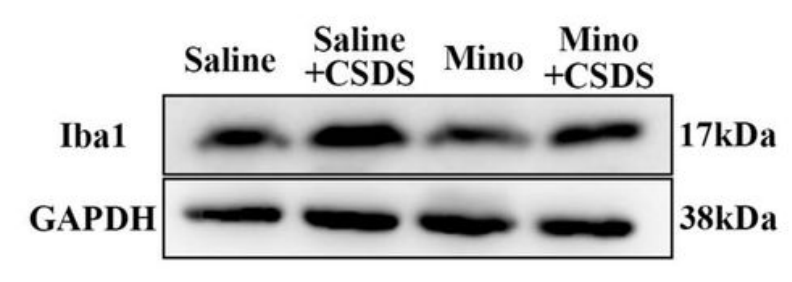

F

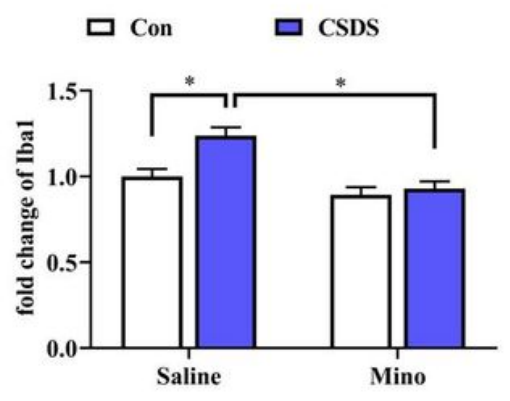

\section{Figure 4}

Minocycline treatment dampened microglial activation in stressed mice. A, Experimental procedure. B, Representative immunofluorescence staining for Ibal in the hippocampus. C, Quantitative analysis of the relative intensity of Iba1. D, Quantitative analysis of the soma size of microglia. E, Representative western blot image for Ibal in the hippocampus. F, Quantitative western blot analysis of the relative intensity of Iba1. $\mathrm{N}=10$ /group. All data are shown as mean $\pm \mathrm{SEM}$. ${ }^{\star} \mathrm{P}<0.05,{ }^{\star} * \mathrm{P}<0.01,{ }^{\star *} \mathrm{P}<0.001$. CSDS: Chronic social defeat stress; Mino: Minocycline. 


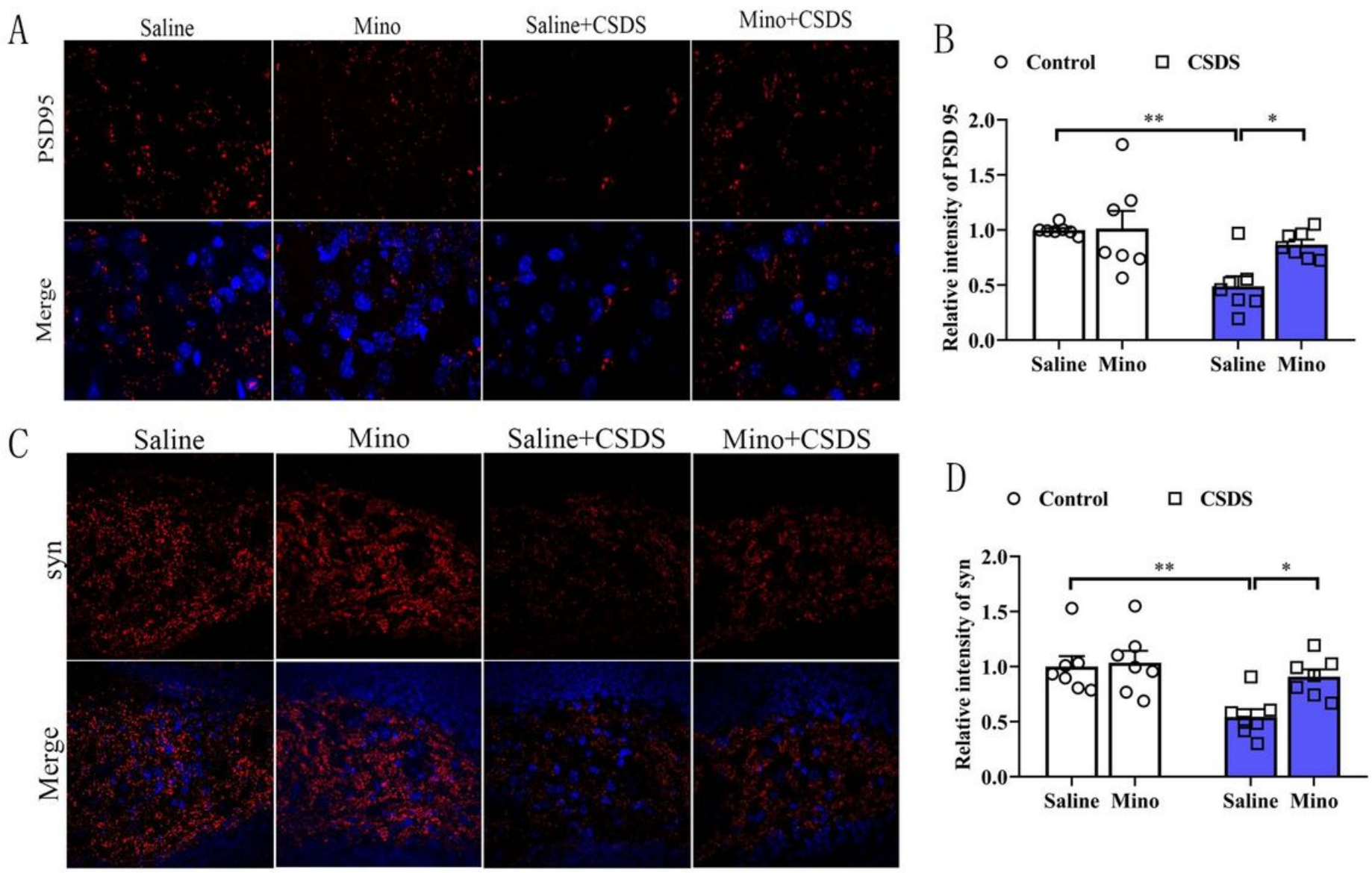

Figure 5

Minocycline treatment alleviated synaptic loss in stressed mice. A, Representative immunofluorescence staining for PSD-95 in the hippocampus. B, Quantitative analysis of the relative intensity of PSD-95. C, Representative immunofluorescence staining for synaptophysin in the hippocampus. D, Quantitative analysis of the relative intensity of synaptophysin. $\mathrm{N}=7 /$ group. All data are shown as mean $\pm \mathrm{SEM}$. ${ }^{\mathrm{P}}<$ $0.05,{ }^{\star} \times P<0.01$. CSDS: Chronic social defeat stress; Mino: Minocycline. 
A

SIT

○ Con $\square$ CSDS
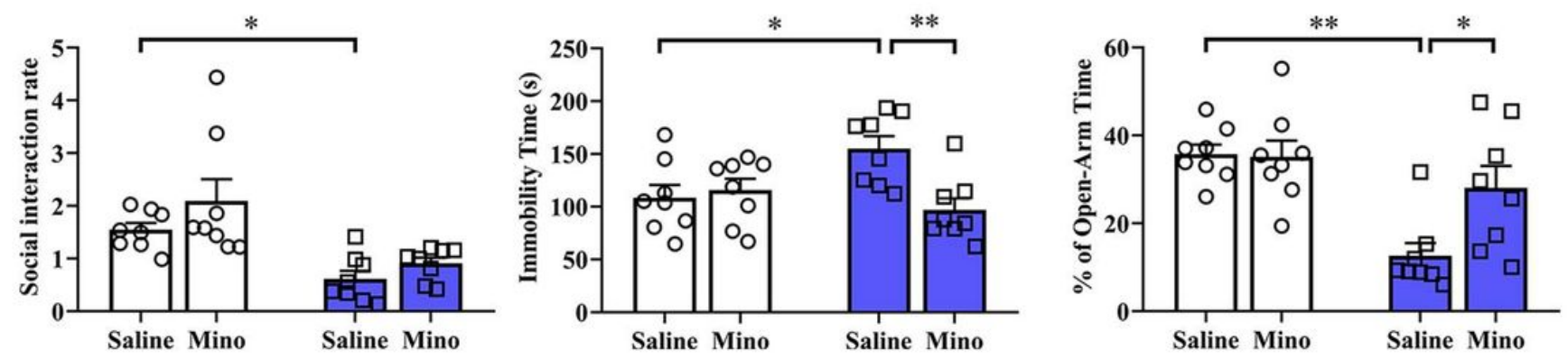

D

E

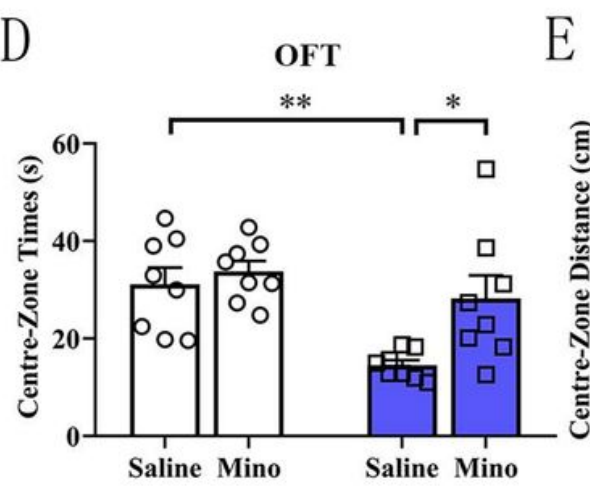

B

FST

C

EPM

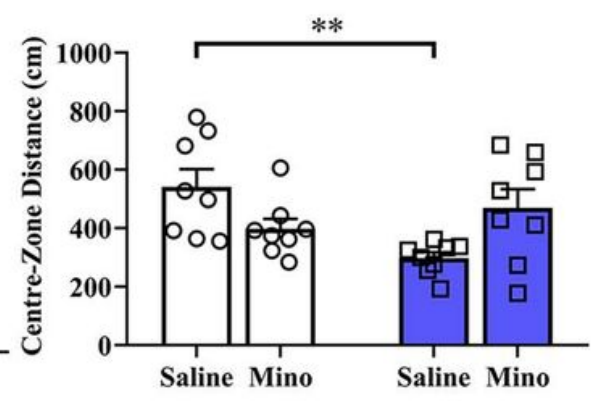

F

OFT

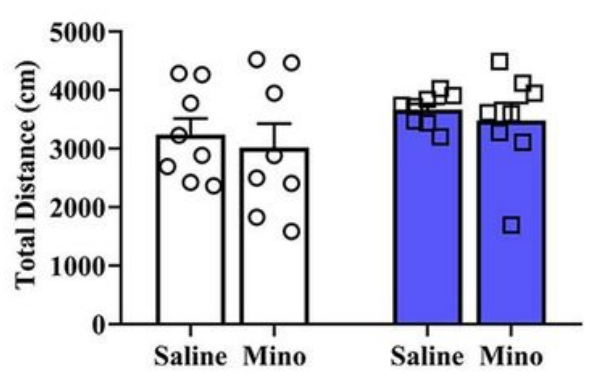

\section{Figure 6}

Minocycline treatment alleviated behavioral impairments in stressed mice. A, Social interaction ratio in the social interaction test. B, Immobility time in the forced swimming test. C, Ratio of time spent in the open arms in the elevated plus maze test. D, Time spent in the center-zone in the open field test. E, Distance traveled in the center-zone in the open field test. F, Total distance traveled in the open field test. $\mathrm{N}=10 /$ group. All data are shown as mean \pm SEM. ${ }^{*} \mathrm{P}<0.05,{ }^{\star \star} \mathrm{P}<0.01$. Con: Control; CSDS: Chronic social defeat stress; Mino: Minocycline. 


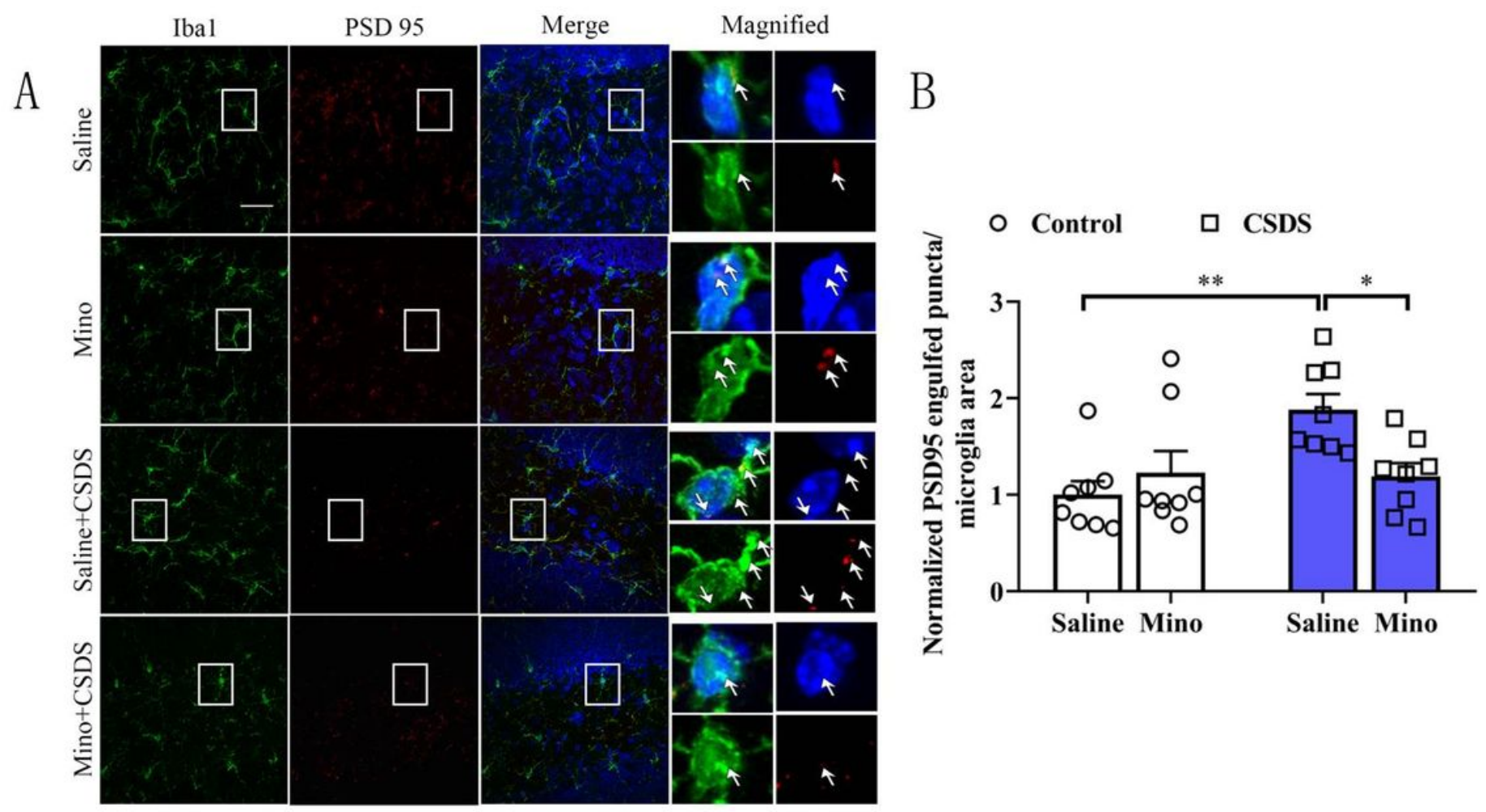

Figure 7

Minocycline treatment reduced abnormal phagocytosis of synapses by microglia in stressed mice. $A$, Representative immunofluorescence staining for PSD-95 co-labeled with Ibal in the hippocampus. B, Quantitative analysis of the PSD-95 engulfed puncta in microglia. $\mathrm{N}=8 /$ group. All data are shown as mean \pm SEM. ${ }^{*} P<0.05,{ }^{*} P<<0.01$. CSDS: Chronic social defeat stress; Mino: Minocycline. 